\title{
Generation of neuroinflammation in human African trypanosomiasis
}

Jean Rodgers, BSc, PhD, Israel Steiner, MD, FRCP, and Peter G. E Kennedy, MD, PhD, DSc

Neurol Neuroimmunol Neuroinflamm 2019;6:e610. doi:10.1212/NXI.0000000000000610
Correspondence

Dr. Rodgers

jean.rodgers@glasgow.ac.uk

\section{Abstract}

Human African trypanosomiasis (HAT) is caused by infection due to protozoan parasites of the Trypanosoma genus and is a major fatal disease throughout sub-Saharan Africa. After an early hemolymphatic stage in which the peripheral tissues are infected, the parasites enter the CNS causing a constellation of neurologic features. Although the CNS stage of HAT has been recognized for over a century, the mechanisms generating the neuroinflammatory response are complex and not well understood. Therefore a better understanding of the mechanisms utilized by the parasites to gain access to the CNS compartment is critical to explaining the generation of neuroinflammation. Contrast-enhanced MRI in a murine model of HAT has shown an early and progressive deterioration of blood-CNS barrier function after trypanosome infection that can be reversed following curative treatment. However, further studies are required to clarify the molecules involved in this process. Another important determinant of brain inflammation is the delicate balance of proinflammatory and counterinflammatory mediators. In mouse models of HAT, proinflammatory mediators such as tumor necrosis factor (TNF)- $\alpha$, interferon (IFN)$\gamma$, and CXCL10 have been shown to be crucial to parasite CNS invasion while administration of interleukin (IL)-10, a counter inflammatory molecule, reduces the CNS parasite burden as well as the severity of the neuroinflammatory response and the clinical symptoms associated with the infection. This review focuses on information, gained from both infected human samples and animal models of HAT, with an emphasis on parasite CNS invasion and the development of neuroinflammation.

From the Institute of Biodiversity (J.R.), Animal Health and Comparative Medicine, University of Glasgow; the Department of Neurology (I.S.), Rabin Medical Center, Campus Beilinson, Petach Tikva, Israel; and the Institute of Infection (P.G.E.K), Immunity and Inflammation, College of Medical, Veterinary and Life Sciences, University of Glasgow. 


\section{Glossary}

BBB = blood-brain barrier; BCSFB = blood-CSF barrier; HAT = human African trypanosomiasis; HBMEC = human brain microvascular endothelial cell; $\mathbf{M M P}=$ matrix metalloproteinases; PTRE = post-treatment reactive encephalopathy; TEER = transendothelial electrical resistance; $\mathbf{T L R}=$ Toll-like receptor; $\mathbf{W B C}=$ white blood cell.

For over a century, human African trypanosomiasis (HAT), also known as sleeping sickness, has been associated with the development of neuroinflammation. ${ }^{1-3}$ However, there is still a significant amount of detail that we do not fully understand regarding the mechanisms that initiate and control the inflammatory response that develops within the CNS as the disease progresses. HAT is endemic in 36 sub-Saharan African countries and results from infection due to the protozoan parasites Trypanosoma brucei (T.b.) gambiense and T.b. rhodesiense. ${ }^{4}$ T.b. gambiense is responsible for around $97 \%$ of HAT cases, while T.b. rhodesiense accounts for the remaining $3 \%$ of the reported cases. The infection is spread via the bite of the tsetse fly vector, so the presence of this insect demarcates the geographical distribution of the infection. ${ }^{4}$ Because of the improved and sustained disease surveillance and control measures, the incidence rate for HAT has dropped significantly in recent years. ${ }^{5}$ Even though the reported case numbers continue to decline, the newly identified presence of asymptomatic carriers ${ }^{6,7}$ together with sociopolitical instability ${ }^{8}$ in many endemic regions raises concerns regarding the feasibility of the World Health Organization's (WHO) target to eliminate HAT as a public health problem by 2020 and interrupt its transmission by $2030{ }^{4}$

\section{HAT disease spectrum}

The 2 subspecies of trypanosome are endemic in discrete geographical regions and produce temporally distinct disease profiles. T.b. gambiense is found in West and Central Africa and follows a chronic progressive course that can persist for months to years before entering the final stages whereas the T.b. rhodesiense variant is present in East and Southern Africa and results in a more acute disease that progresses rapidly with an average duration of only weeks to months. ${ }^{4,9,10}$ The geographical distinction between these 2 disease forms is becoming less pronounced as the topographical range of the different subspecies is gradually moving closer and could soon overlap due to the movement of both people and domestic cattle, which act as reservoir hosts for T.b. rhodesiense. ${ }^{11}$ T.b. gambiense and T.b. rhodesiense are indistinguishable by microscopy and can only be differentiated using molecular methods that are not commonly available in the African field. Therefore, if the range of these 2 parasites were to merge, this would confound current diagnostic approaches and could lead to administration of inappropriate chemotherapy. ${ }^{12}$

The clinical progression of HAT has been described in detail elsewhere. ${ }^{4,5,9,10}$ Briefly, after the tsetse bite, the trypanosomes proliferate at the wound site, which can result in the formation of a nonsuppurative but painful, indurated lesion known as a trypanosomal chancre. ${ }^{4}$ HAT is traditionally described as developing through 2 stages; the early or hemolymphatic stage where the trypanosomes spread from the initial bite site and multiply in the blood, lymphatics, and peripheral tissues, and the late or encephalitic stage where the parasites migrate into the CNS, producing a range of neurologic manifestations. ${ }^{4,9}$ For many years, the infection was considered fatal if untreated; however, there is growing evidence that some patients affected by T.b. gambiense remain asymptomatic and may self-cure. ${ }^{7}$

Clinical signs suggestive of neurologic involvement such as the presence of an altered gait, incontinence, tremors, cranial nerve palsy, or a reduced Glasgow Coma Scale have been recorded during early stage infections in human patients, ${ }^{13}$ indicating an apparent disparity between parasitological disease progression and the evolution of neurologic signs of infection. The transition from hemolymphatic to encephalitic stage is therefore insidious with no clear clinical indication to distinguish between the 2 disease stages.

\section{Diagnosis and disease staging}

Although clinical signs are suggestive of trypanosome infection, they cannot be considered as diagnostic of the disease. Many of these symptoms are found in infections such as malaria, HIV, and tuberculosis that coexist in the same geographical areas as HAT. ${ }^{10,14}$ Consequently, diagnosis is generally a multistepped process, relying on both clinical presentation and laboratory testing to identify the parasites through direct detection or serologic approaches. ${ }^{4}$

Once HAT is confirmed, accurate disease staging is of vital importance in directing the appropriate chemotherapy because current therapy for late (encephalitic) stage disease is more toxic than that of early stage disease. WHO guidelines classify patients with more than 5 white blood cells (WBCs) $/ \mu \mathrm{L}$ and/or trypanosomes in their CSF as in the encephalitic stage. ${ }^{4}$ This CSF WBC criterion, however, is not universally accepted ${ }^{5,9}$ and there are no reliable biomarkers to distinguish between the 2 disease stages. Several molecules have been trialed with varying levels of success (table) ${ }^{15-21}$ Research in this area is ongoing, but the current lack of an accurate method to provide a reliable comparator means that researchers must depend on the debatable WHO guidelines making this task more challenging. ${ }^{22,23}$ 
Table Potential biomarkers of CNS-stage trypanosome infections

\begin{tabular}{|c|c|c|}
\hline Biomarker/panel & Methodology & Trypanosome species \\
\hline CXCL13, CXCL10, IgM ${ }^{20}$ & CSF-immunoassay & T.b. rhodesiense \\
\hline CXCL13, CXCL10, MMP-920 & CSF-immunoassay & T.b. rhodesiense \\
\hline CXCL10, CXCL8, H-FABP ${ }^{17}$ & CSF-ELISA and bead suspension array & T.b. gambiense \\
\hline Neopterin, 5-hydroxytryptophan ${ }^{21}$ & CSF-metabolomics & T.b. gambiense \\
\hline Ornithine, aminododecanoic acid ${ }^{21}$ & Plasma-metabolomics & T.b. gambiense \\
\hline Neopterin $^{18}$ & CSF-ELISA & T.b. gambiense \\
\hline ICAM-1, MMP-9 $^{16}$ & CSF-bead suspension array & T.b. gambiense \\
\hline Osteopontin, $\beta$-2-microglobulin 19 & CSF-ELISA & T.b. gambiense \\
\hline CXCL10, lipocalin 2, SLPI ${ }^{15}$ & CSF-ELISA & T.b. gambiense, T.b. rhodesiense \\
\hline
\end{tabular}

Abbreviations: $\mathrm{H}-\mathrm{FABP}$ = heart-fatty acid binding protein; ICAM = intercellular adhesion molecule; MMP = matrix metalloproteinase; SLPI = secretory leukocyte peptidase inhibitor.

\section{Chemotherapy}

Treatment of HAT is complex because of a lack of safe drugs that are effective against both stages and variants of the disease. Pentamidine and suramin are used to treat stage 1 T.b. gambiense and T.b. rhodesiense infections, respectively. When the disease has progressed to the encephalitic stage, a combination of nifurtimox and eflornithine is the first-line treatment for T.b. gambiense infections while only the trivalent arsenical melarsoprol can be used for stage 2 T.b. rhodesiense disease. ${ }^{4}$ All of these regimens rely on parenteral administration of the drugs involved. Recently an orally deliverable drug, fexinidazole, has been trialed with some success in the treatment of stage 1 and stage 2 of T.b. gambiense infections and may replace the currently recommended treatment protocols for this disease variant. ${ }^{24}$ Details of these chemotherapy regimens have been fully described elsewhere., ${ }^{4,24}$

\section{Neuropathogenesis}

\section{Postmortem examination}

There are limited historical reports describing the neuropathologic changes seen in patients after fatal T.b. gambiense and T.b. rhodesiense infections. The earliest of these reports, made by Mott ${ }^{1}$ in 1899, describes the neuropathologic changes in 2 fatal cases of "Negro Lethargy". This study was performed prior to trypanosomes being identified as the etiological agents of "sleeping sickness;" however, the clinical and neuropathologic signs described in the 2 Congolese patients clearly implicate T.b. gambiense infection. In both cases, Mott describes the development of a diffuse meningoencephalomyelitis withlymphocytes infiltrating along the blood vessels and into the substance of the brain. This neuroinflammatory reaction is now a recognized characteristic of CNS-stage HAT and has since been noted in additional descriptions produced from fatal cases. $^{2,25-29}$ The inflammatory cell infiltrate is comprised predominantly of mononuclear lymphocytes together with a large number of plasma cells. ${ }^{2,27,29}$ Morular or Mott cells are also a common feature but cannot be considered as pathognomonic of HAT. ${ }^{2,27,28}$ Marked astrocyte activation and microglial reactivity accompanies the inflammatory cell infiltration and is especially prominent in the white matter. ${ }^{28}$ Even in cases where the neuroinflammatory reaction is severe, there appears to be a significant sparing of the nervous tissue and demyelination is generally minimal. ${ }^{27-29}$ Indications of inflammation in the ventricles and choroid plexus were noted on only a few occasions by Mott $^{2}$ and Adams; ${ }^{27}$ however in Calwell's investigation of T.b. rhodesiense infections, lymphocyte infiltration, fibrosis, and edema were observed in the choroid plexus in 7 of the 8 cases examined, although these patients had received various trypanocidal drugs. ${ }^{28}$ In summary, the predominant neuropathologic changes associated with CNS-stage HAT are most apparent in the white matter regions. These changes are characterized by the presence of a diffuse, lymphocytic-plasmacytic meningoencephalitis of varying severity accompanied by activation of astrocytes and microglia; however, only slight demyelination occurs and there is limited neuronal involvement.

In some instances, patients experience a severe adverse reaction to treatment with trypanocidal drugs, melarsoprol in particular, resulting in the development of a posttreatment reactive encephalopathy (PTRE) ${ }^{27}$ The neuropathologic lesions exhibited by these patients present as a significant exacerbation of the neuroinflammatory reaction described above with severe perivascular cuffing and inflammatory cell infiltration of the brain parenchyma. This reaction can progress in severity and features associated with tentorial herniation and acute hemorrhagic leukoencephalopathy have been reported. ${ }^{27}$

\section{Animal models}

Reports describing the neuropathologic changes in human patients are uncommon so most of our knowledge regarding neuropathogenesis has been derived from rodent and primate 
models of the infection. ${ }^{30-34}$ The neuropathologic response generated in these animal models is largely similar to the pattern described in fatal cases of HAT with the most pronounced reaction seen in the white matter and characterized by glial cell activation accompanied by lymphocyte, macrophage, and plasma cell invasion of the perivascular space and spreading to the brain parenchyma in advanced disease. The neuroinflammatory response generally remains mild with severe neuroinflammation and encephalitis most commonly reported after subcurative drug treatment. ${ }^{33}$ This finding emphasizes the relationship between exacerbation of the neuroinflammatory reaction and trypanocidal drug administration and is most akin to the situation encountered when patients develop the PTRE. The mechanisms precipitating the PTRE remain unclear. Many theories have been suggested including drug toxicity, the release of parasite antigens within the CNS, immune complex deposition, autoimmunity, and a robust immune response to residual parasites within the CNS after drug treatment. ${ }^{35}$ There is some variation in the neuropathogenesis of the disease between species. Inflammatory lesions and trypanosomes are commonly found in the choroid plexus in dogs with little infiltration of the meninges. In contrast, the most severe response is seen in the meninges in cattle with a less pronounced and more focal reaction present in the choroid plexus. ${ }^{36}$

By combining animal models of HAT with contemporary techniques such as confocal and multiphoton microscopy and MRI details of the mechanisms generating the neuroinflammatory reaction and facilitating trypanosome transmigration into the CNS are beginning to emerge. However to enter the brain parenchyma, inflammatory cells and parasites must first negotiate the blood-CNS barriers.

\section{Blood-CNS barriers}

Since optimal brain function is highly reliant on maintaining a specific microenvironment, all influx and efflux between the central compartment and the periphery are tightly controlled. Therefore, the CNS is effectively isolated from the peripheral systems by the presence of specialized barriers, situated at 3 main sites within the CNS, which regulate the passage of cells and molecules between the nervous tissue and the blood. There are several different pathways through these barriers that can be utilized to access the brain and these are described in detail elsewhere. ${ }^{37}$ The 3 barrier forms include the classical parenchymal blood-brain barrier (BBB), which separates the vasculature from the CNS parenchyma; the blood-CSF barrier (BCSFB), which forms an interface between the choroid plexus and the ventricular CSF; and the arachnoid barrier, which is found in the middle layer of the meninges and acts as a barrier between the dura and the subarachnoid CSF. Morphologically, differences exist between these barriers with the parenchymal $\mathrm{BBB}$ showing the greatest complexity and being the most prevalent of the 3 blood-CNS interfaces. ${ }^{37}$ The parenchymal $\mathrm{BBB}$ is formed by several cell types including brain microvascular endothelial cells that are joined by complex tight junctions and pericytes, which partially surround the vessels and are disseminated along the cerebral capillaries (figure). Both are embedded in the endothelial basement membrane. This is bordered by a CSF-filled perivascular space and further bounded by the parenchymal basement membrane incorporating the glial limitans perivascularis, comprised of astrocyte end-feet. Neurons and microglial cells are found in close apposition to these components (figure). These cell types interact to form the neurovascular units that, together with the basement membranes, constitute the parenchymal BBB. ${ }^{37}$ The function of the blood-CNS barriers is dynamic, responding to a host of signals originating in both the peripheral and the central compartments, with barrier dysfunction having a major impact in a wide range of disease etiologies. ${ }^{37}$

\section{Transmigration of trypanosomes into the CNS}

In vitro $\mathrm{BBB}$ models have been developed comprising cultured human brain microvascular endothelial cells (HBMECs) grown on Transwell inserts producing an upper and lower compartment mirroring the luminal and abluminal sides of the brain microvasculature. The HBMECs express a range of markers and exhibit characteristics associated with $\mathrm{BBB}$ endothelial cells in vivo. ${ }^{38}$ When trypanosomes are introduced into the luminal side of the model, they transmigrate across the HBMEC layer producing only a transient reduction in transendothelial electrical resistance (TEER) suggesting that trypanosome transmigration does not trigger an irreversible loss of barrier function. ${ }^{38}$ Inhibition of several enzymes including phospholipase $\mathrm{C}$, protein kinase, and the parasite cysteine protease brucipain (i.e., cathepsin-L) or chelation of $\mathrm{Ca}^{2+}$ prevents trypanosome transmigration and precludes the concomitant reduction in TEER. ${ }^{39}$ In addition, blocking the G protein-coupled receptor, protease-activated receptor-2, shown to trigger calcium-mediated transmembrane signaling in endothelial cells, and previously implicated in a variety of neuroinflammatory conditions, can prevent parasite transmigration across the in vitro barrier. ${ }^{40}$ These findings not only show that parasites have the ability to traverse the $\mathrm{BBB}$ endothelial cell layer but also suggest that calcium signaling could play an important role in trypanosome invasion of the CNS (figure).

Despite these in vitro findings, microscopic examinations of brain sections prepared from fatal HAT cases have detected trypanosomes infrequently within the CNS. In a study of 6 postmortem cases of T.b. rhodesiense infection, Manuelidis et al. $^{29}$ found only a single parasite in the numerous sections examined while Calwell ${ }^{28}$ failed to detect trypanosomes in the CNS in a series of 17 patients. Mott ${ }^{2}$ also failed to detect intact parasites in the brain tissue in T.b. gambiense patients, but reported trypanosomes either confined to blood vessels or the presence of possible degenerate forms in the perivascular infiltrates. Several hypotheses have been mooted to explain the apparent absence of trypanosomes from the brain tissue including the rapid degradation of parasites after death of the host or clearance as a result of trypanocidal chemotherapy. ${ }^{27,28}$

In contrast, trypanosomes are frequently seen within the brain parenchyma in rodent models during both acute ${ }^{41,42}$ and chronic $^{31,32,43,44}$ infections and have therefore facilitated the 


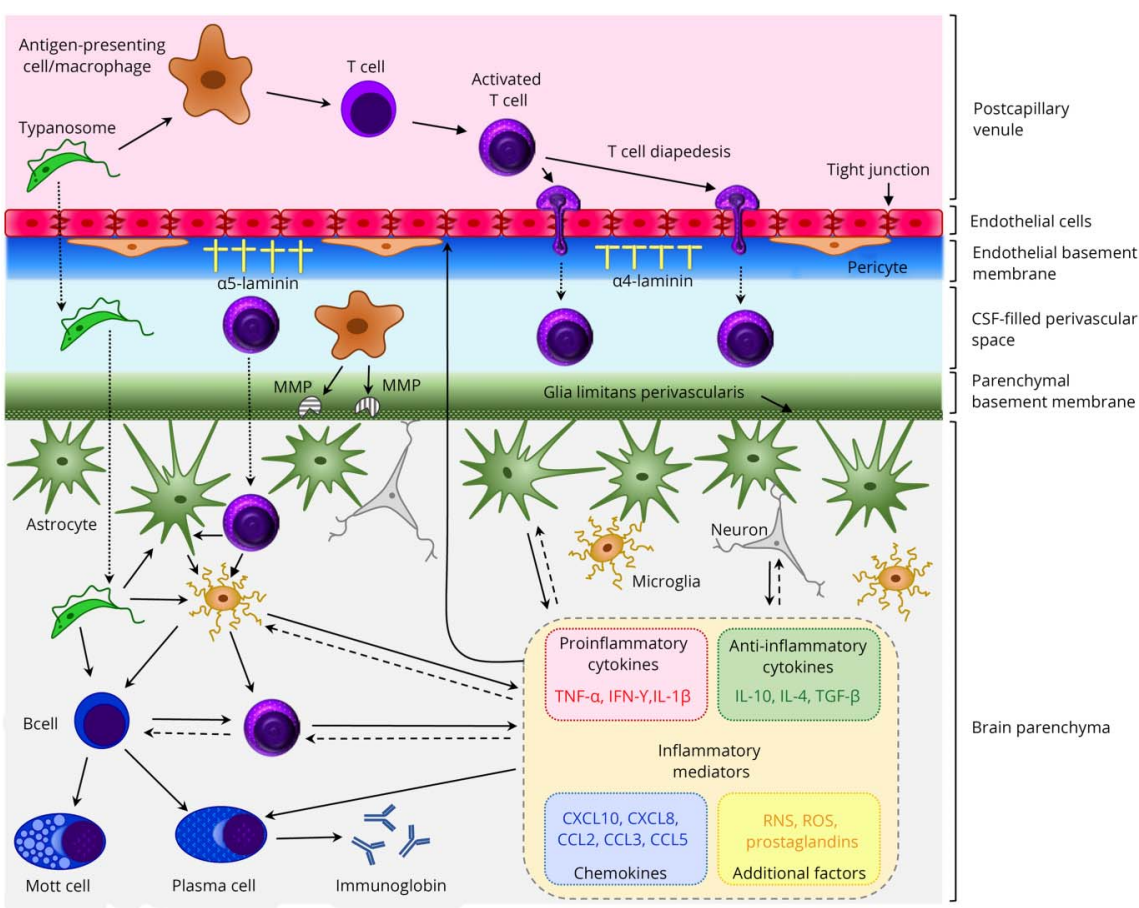

In the healthy individual, a limited number of $T$ cells enter the brain to carry out immune surveillance. As HAT advances, lymphocytes and parasites migrate from postcapillary venules across the BBB to enter the brain parenchyma. The mechanisms facilitating this progression and those controlling the ensuing neuroinflammatory reaction are not fully understood; however, some aspects have been elucidated. The production of the cysteine protease, brucipain, by the parasites increases their ability to migrate across the BBB endothelial cell layer. After activation in the peripheral compartment, T-cell diapedesis through the endothelial cell layer of the barrier can occur using both paracellular and transcellular routes. On breaching the endothelial cell layer, both T-cells and trypanosomes must penetrate the endothelial basement membrane. This is dependent on the laminin subtypes present. Areas where a4 laminin predominates allow transmigration while a5 laminin-rich regions inhibit crossing into the perivascular space. Before reaching the brain parenchyma, T cells and parasites must traverse the parenchymal basement membrane. This step appears to be dependent on a number of factors including the presence of IFN-y and the production of MMPs, most likely by perivascular macrophages, which disengage the astrocyte end-feet from the glial limitans allowing the cells to enter the brain. The T cells can then interact with resident microglia, astrocytes, or additional lymphocytes that can each produce a range of inflammatory mediators. The overall balance of these mediators is critical in controlling the neuroinflammatory response and BBB integrity and may prompt clinical manifestations of the disease including pyrexia, cachexia, and sleep disturbances. $\mathrm{BBB}=$ blood-brain barrier; HAT = human African trypanosomiasis; MMP = matrix metalloproteinases.

investigation of trypanosome invasion of the CNS. The established paradigm suggests that the parasites use a multistepped process to colonize the brain parenchyma. In the first instance, trypanosomes appear in the stroma of the choroid plexus and the circumventricular organs. In both of these areas, the blood vessels are fenestrated, which could allow passage of the parasites to the abluminal side. However, it is unlikely that these fenestrations alone provide a suitable channel for trypanosome traversal because of their restrictive size and a much more complex interplay between the parasites and the epithelial cells is probable to facilitate transmigration. Furthermore, to pass from the stroma of the choroid plexus into the ventricular CSF, the trypanosomes must deal with the BCSFB, formed by the choroid plexus epithelial cells. ${ }^{45,46}$ The ability of trypanosomes to survive within the choroid plexus and the CSF remains equivocal ${ }^{47}$ and the cyclical nature of the parasite burden in these areas closely follows fluctuations in parasitemia, which has been suggested as a replenishing source of the trypanosome population within these compartments. ${ }^{48}$ Additional studies utilizing freeze fracture electron microscopy have proposed that parasites persist as a viable population residing between the cell layers of the pia mater thereby avoiding the apparently hostile environs of the CSF. ${ }^{47}$
The effects of trypanosome infection on blood-CNS barrier function have been investigated using in vivo disease models. Initial studies suggested that trypanosome infection reduced barrier function during the later stages of the disease as peripherally injected dye could be detected within the brain parenchyma. ${ }^{44}$ These findings remained equivocal as no changes in the presence of occludin and zonula occludens-1 or leakage of fibrinogen and IgG into the brain could be detected in a similar disease model. ${ }^{43}$ However, a more recent study utilizing contrast-enhanced MRI in conjunction with a murine HAT model has described a gradual and progressive deterioration in barrier integrity beginning prior to the onset of CNS-stage infection. ${ }^{30}$ Whether this is a direct effect of the trypanosomes or a symptom of the peripheral inflammatory reaction remains to be explained. Further evidence suggests that this reduction in barrier function is reversible and can be restored after curative chemotherapy. $^{49}$

Although it would seem logical to assume that the parasites follow a step-wise dissemination from the CSF to the brain parenchyma, studies by Masocha et al. ${ }^{50}$ suggest that the trypanosomes gain entry to the neuropil via a hematogenous route rather than originating from the CSF compartments. In fact, 
using confocal microscopy, T. $b$. brucei has been detected throughout the cortex, septum, corpus callosum, striatum, thalamus, and traversing blood vessels in the hypothalamus at 9-day postinfection using double immunofluorescence to label both trypanosomes and brain microvascular endothelial cells in a rat model of $\mathrm{HAT}^{42}$ This early CNS invasion has also been demonstrated by intravital microscopy to reveal both T. $b$. brucei and T.b. rhodesiense parasites, genetically modified to express a fluorescent marker, within the parietal cortex 5 hours after high-dose IV infections in $\mathrm{C} 57 \mathrm{Bl} / 6$ mice. ${ }^{41}$ However, the relevance of these findings of early and widespread CNS invasion to the situation found in HAT remains equivocal. It is therefore clear that further work is warranted to establish more accurate temporal-spatial kinetics to comprehend exactly when and where trypanosomes enter the CNS.

\section{Influence of immune factors}

The mechanisms employed by the parasites to circumvent the CNS barriers and generation of the associated neuroinflammation are multifactorial and not fully understood. However, evidence is emerging to suggest that the innate immune response plays an important role in HAT pathogenesis. ${ }^{51}$ Mice deficient in Toll-like receptor (TLR)-9, an innate signaling molecule, exhibit lower numbers of $\mathrm{T}$ cells in the CNS compared to their wild-type counterparts after trypanosome infection. ${ }^{52}$ MyD88 is an intracellular adaptor protein required for maintaining the downstream TLR signal transduction pathways responsible for generating innate inflammation as well as priming the adaptive immune response. Reduced numbers of T cells were found in the brain in infected MyD88 knockout mice, accompanied by a corresponding increase in parasite numbers that were not present in TLR-9 knockout mice. Therefore, taken together these findings suggest that pathways other than TLR-9, involving MyD88 signaling, may play a role in controlling the trypanosome population within the brain. ${ }^{52}$ However, ablation of IL-1R, IL-18, TLR-2, or TLR4, which all signal through MyD88, did not show alterations in $\mathrm{T}$ cell or parasite levels in the brain. ${ }^{52}$ TLR signaling is closely involved with the control of cytokine and chemokine expression and these important immunologic moderators are known to play key roles in the progression of trypanosome infections. ${ }^{51}$ In summary, TLR signaling has been shown to stimulate the expression of TNF- $\alpha$ and type- 1 IFN that could initiate a cascade of events culminating in trypanosome and $\mathrm{T}$-cell transmigration into the CNS while additional mediators within the brain exert control over the expansion of the parasite population residing within the CNS.

Another pathway recently implicated in the generation of the neuroinflammatory reaction to trypanosome infection is the kynurenine pathway. ${ }^{53,54}$ This pathway is fundamental in the oxidation of tryptophan, produces several neurotoxic and neuroprotective catabolites, and is important in influencing both the innate and adaptive immune response. ${ }^{55}$ In a murine model of HAT, inhibition of kynurenine 3-monooxygenase, a key enzyme in this pathway, resulted in a significant reduction in the severity of the neuroinflammatory reaction exhibited during the late CNS stage of the infection. ${ }^{54}$ Activation of this pathway has been found through liquid chromatography-mass spectrometry analysis of CSF samples from patients with both early and late-stage $\mathrm{HAT}^{53}$ In these patients, significant increases in kynurenine levels, accompanied by decreased tryptophan concentrations, were observed in infected individuals compared to uninfected controls. ${ }^{53}$ An association was also found between tryptophan catabolism and increased CSF IL-6 concentrations, providing further evidence to support a role for this pathway in the neuropathogenesis of HAT. Changes in the concentration of kynurenine pathway metabolites in the CSF were also identified in a metabolomics study, designed to detect potential biomarkers for late-stage HAT, although these changes were small. ${ }^{21}$

In a murine model of HAT, several cytokines, including TNF$\alpha$ and IFN- $\gamma$, have been associated with the presence of a moderate to severe neuroinflammatory reaction, while IL-10 and IL-6 were found at higher levels early after infection when only a mild neuroinflammation was present. ${ }^{56}$ The importance of these cytokines in CNS-stage infections was further demonstrated through finding that $\mathrm{T}$ cells and trypanosomes arrest in the perivascular space and do not progress through the parenchymal basement membrane to enter the neuropil in IFN- $\gamma$ or IFN- $\gamma$ receptor knockout mice (figure). This phenomenon was also seen in IL12p40 knockout mice that cannot initiate IFN- $\gamma$ secretion. ${ }^{50}$ Furthermore, systemic administration of IL-10 in a late-stage mouse model of HAT improved the clinical response of the animals to the infection compare to untreated animals. ${ }^{57}$ IL-10 treatment also resulted in lower plasma levels of IFN- $\gamma$ and TNF- $\alpha$, accompanied by a reduction in the severity of the neuroinflammatory reaction and parasite burden in the brain, which may be a consequence of the reduced presence of IFN- $\gamma .{ }^{57}$

The close association between T-cell and parasite transmigration into the CNS after trypanosome infection has been further demonstrated. In a similar pattern to T-cell transmigration, vascular basement membrane regions expressing laminin $a 4$ were shown to be permissive to parasites while areas where laminin $\alpha 5$ predominated inhibited parasite crossing. ${ }^{50}$ In the CNS, laminin $\alpha 4$ regions are widespread along the endothelial basement membrane whereas laminin a5 areas occur less frequently. However, to facilitate passage into the neuropil, the cells and parasites must also traverse the parenchymal basement membrane (figure). Activation of matrix metalloproteinases (MMPs)-2 and -9 cleave the dystroglycan receptors from the astrocyte end-feet causing them to disengage from the parenchymal basement membrane, compromising the integrity and allowing transmigration into the brain. ${ }^{58}$ Increased MMP activity, together with decreased activation of tissue inhibitors of MMPs at the BCSFB in vitro, has been associated with TNF- $a$ expression, leading to increased barrier permeability, ${ }^{59}$ again implicating a pivotal role for this molecule in the progression of HAT. Furthermore, critical roles for both laminins and MMPs, and their integral connection with cytokine and chemokine 
expression, have been identified in the progression of experimental autoimmune encephalomyelitis, ${ }^{45}$ and changes in the expression pattern of MMPs have been detected in rodent models of HAT. ${ }^{60}$

\section{Discussion}

Although many advances have been made with regard to understanding the pathogenesis of HAT, there are still numerous unanswered questions and areas where our current knowledge remains incomplete. A fuller understanding of the pathways involved in the disease process will provide insights to improve management strategies and chemotherapy options. Therefore, the continued application of contemporary analytics, combined with both in vivo and in vitro approaches to model the disease, together with improved access to human samples is necessary to achieve a greater understanding of this ancient and complex parasitic infection.

\section{Study funding \\ Funded by University of Glasgow}

\section{Disclosure}

The authors report no dislosures. Go to Neurology.org/NN for full disclosures.

\section{Appendix Authors}

\begin{tabular}{|c|c|c|c|}
\hline Name & Location & Role & Contribution \\
\hline $\begin{array}{l}\text { Jean Rodgers } \\
\text { BSc, PhD }\end{array}$ & $\begin{array}{l}\text { Institute of Biodiversity, } \\
\text { Animal Health and } \\
\text { Comparative Medicine, } \\
\text { University of Glasgow, } \\
\text { Bearsden Road, Glasgow, UK }\end{array}$ & Author & $\begin{array}{l}\text { Drafting and } \\
\text { revision of } \\
\text { manuscript }\end{array}$ \\
\hline $\begin{array}{l}\text { Israel Steiner } \\
\text { MD }\end{array}$ & $\begin{array}{l}\text { Department of Neurology, } \\
\text { Rabin Medical Center, } \\
\text { Campus Beilinson, } 49100 \\
\text { Petach Tikva, Israel }\end{array}$ & Author & $\begin{array}{l}\text { Commented } \\
\text { and added to } \\
\text { draft }\end{array}$ \\
\hline $\begin{array}{l}\text { Peter G E } \\
\text { Kennedy MD, } \\
\text { PhD, DSc }\end{array}$ & $\begin{array}{l}\text { Institute of Infection, } \\
\text { Immunity and } \\
\text { Inflammation, College of } \\
\text { Medical, Veterinary and Life } \\
\text { Sciences, University of } \\
\text { Glasgow, Glasgow, UK }\end{array}$ & Author & $\begin{array}{l}\text { Commented } \\
\text { and added to } \\
\text { draft }\end{array}$ \\
\hline
\end{tabular}

\section{Publication history}

Received by Neurology: Neuroimmunology \& Neuroinflammation April 15, 2019. Accepted in final form July 29, 2019.

\section{References}

1. Mott FW. The changes in the central nervous system of two cases of Negro Lethargy: sequel to Dr. Manson's clinical report. Br Med J 1899;2:1666-1669.

2. Mott FW. Reports of sleeping sickness commission. No. VII. Histological Observations on Sleeping Sickness and Other Trypanosome Infections. London: John Bale, Sons \& Danielsson, Ltd; 1906.

3. Mott FW. Observations on the brains of men and animals infected with various forms of trypanosomes. Preliminary note. Proceedings of the Royal Society of London Series B-Containing Papers of a Biological Character. 1905;76:235-242.
4. WHO. Control. Surveillance of Human African Trypanosomiasis: Report of a WHO Expert Committee. Geneva: WHO technical report series; 2013:984.

5. Kennedy PGE, Rodgers J. Clinical and neuropathogenetic aspects of human African trypanosomiasis. Front Immunol 2019;10:39.

6. Casas-Sanchez A, Acosta-Serrano A. Skin deep. Elife 2016;5:e21506.

7. Jamonneau V, Ilboudo H, Kabore J, et al. Untreated human infections by Trypanosoma brucei gambiense are not 100\% fatal. PLoS Negl Trop Dis 2012;6:e1691.

8. Berrang-Ford L, Lundine J, Breau S. Conflict and human African trypanosomiasis. Soc Sci Med 2011;72:398-407.

9. Kennedy PG. Human African trypanosomiasis of the CNS: current issues and challenges. J Clin Invest 2004;113:496-504.

10. Kennedy PG. Clinical features, diagnosis, and treatment of human African trypanosomiasis (sleeping sickness). Lancet Neurol 2013;12:186-194.

11. Batchelor NA, Atkinson PM, Gething PW, et al. Spatial predictions of Rhodesian Human African Trypanosomiasis (sleeping sickness) prevalence in Kaberamaido and Dokolo, two newly affected districts of Uganda. PLoS Negl Trop Dis 2009;3:e563.

12. Picozzi K, Fèvre EM, Odiit M, et al. Sleeping sickness in Uganda: a thin line between two fatal diseases. BMJ 2005;331:1238-1241.

13. MacLean L, Reiber H, Kennedy PG, Sternberg JM. Stage progression and neurological symptoms in Trypanosoma brucei rhodesiense sleeping sickness: role of the CNS inflammatory response. PLoS Negl Trop Dis 2012;6:e1857.

14. Atouguia JLM, Kennedy PGE. Neurological aspects of human African trypanosomiasis. In: Davies LE, Kennedy PGE, editors. Infectious Diseases of the Nervous System. Oxford: Butterworth-Heinemann; 2000:321-372.

15. Amin DN, Ngoyi DM, Nhkwachi GM, et al. Identification of stage biomarkers for human African trypanosomiasis. Am J Trop Med Hyg 2010;82:983-990.

16. Hainard A, Tiberti N, Robin X, et al. Matrix metalloproteinase-9 and intercellular adhesion molecule 1 are powerful staging markers for human African trypanosomiasis. Trop Med Int Health 2011;16:119-126.

17. Hainard A, Tiberti N, Robin X, et al. A combined CXCL10, CXCL8 and H-FABP panel for the staging of human African trypanosomiasis patients. PLoS Negl Trop Dis 2009;3:e459.

18. Tiberti N, Hainard A, Lejon V, et al. Cerebrospinal fluid neopterin as marker of the meningo-encephalitic stage of Trypanosoma brucei gambiense sleeping sickness. PLoS One 2012;7:e40909.

19. Tiberti N, Hainard A, Lejon V, et al. Discovery and verification of osteopontin and Beta-2-microglobulin as promising markers for staging human African trypanosomiasis. Mol Cell Proteomics 2010;9:2783-2795.

20. Tiberti N, Matovu E, Hainard A, et al. New biomarkers for stage determination in Trypanosoma brucei rhodesiense sleeping sickness patients. Clin Transl Med 2013;2:1.

21. Vincent IM, Daly R, Courtioux B, et al. Metabolomics identifies multiple candidate biomarkers to diagnose and stage human African trypanosomiasis. PLoS Negl Trop Dis 2016; 10:e005140.

22. Kennedy PG. Diagnosing central nervous system trypanosomiasis: two stage or not to stage? Trans R Soc Trop Med Hyg 2008;102:306-307.

23. Njamnshi AK, Gettinby G, Kennedy PGE. The challenging problem of disease staging in human African trypanosomiasis (sleeping sickness): a new approach to a circular question. Trans R Soc Trop Med Hyg 2017;111:199-203.

24. Mesu V, Kalonji WM, Bardonneau C, et al. Oral fexinidazole for late-stage African Trypanosoma brucei gambiense trypanosomiasis: a pivotal multicentre, randomised, non-inferiority trial. Lancet 2018;391:144-154.

25. Mott FW. The comparative neuropathology of trypanosome and spirochate infections, with a resume of our knowledge of human trypanosomiasis. Pro R Soc Med 1911;4:1-40.

26. Poltera AA, Owor R, Cox JN. Pathological aspects of human African trypanosomiasis (HAT) in Uganda. A post-mortem survey of fourteen cases. Virchows Arch A Pathol Anat Histol 1977;373:249-265.

27. Adams JH, Haller L, Boa FY, Doua F, Dago A, Konian K. Human African trypanosomiasis ( T. b. gambiense ): a study of 16 fatal cases of sleeping sickness with some observations on acute reactive arsenical encephalopathy. Neuropatho Appl Neurobiol 1986;12:81-94.

28. Calwell HG. The pathology of the brain in Rhodesian trypanosomiasis. Trans R Soc Trop Med Hyg 1937;30:611-624.

29. Manuelidis EE, Robertson DH, Amberson JM, Polak M, Haymaker W. Trypanosoma rhodesiense encephalitis. Clinicopathological study of five cases of encephalitis and one of mel B hemorrhagic encephalopathy. Acta Neuropathol 1965;5:176-204.

30. Rodgers J, Bradley B, Kennedy PGE. Delineating neuroinflammation, parasite CNS invasion, and blood-brain barrier dysfunction in an experimental murine model of human African trypanosomiasis. Methods 2017;127:79-87.

31. Fink E, Schmidt H. Meningoencephalitis in chronic Trypanosoma brucei rhodesiense infection of the white mouse. Tropenmed Parasitol 1979;30:206-211.

32. Poltera AA, Hochmann A, Rudin W, Lambert PH. Trypanosoma brucei brucei: a model for cerebral trypanosomiasis in mice-an immunological, histological and electronmicroscopic study. Clin Exp Immunol 1980;40:496-507.

33. Schmidt H. The pathogenesis of trypanosomiasis of the CNS. Studies on parasitological and neurohistological findings in trypanosoma rhodesiense infected vervet monkeys. Virchows Arch A Pathol Anat Histopathol 1983;399:333-343.

34. Ouwe-Missi-Oukem-Boyer O, Mezui-Me-Ndong J, Boda C, et al. The vervet monkey (Chlorocebus aethiops) as an experimental model for Trypanosoma brucei gambiense human African trypanosomiasis: a clinical, biological and pathological study. Trans R Soc Trop Med Hyg 2006;100:427-436.

35. Hunter CA, Jennings FW, Adams JH, Murray M, Kennedy PG. Subcurative chemotherapy and fatal post-treatment reactive encephalopathies in African trypanosomiasis. Lancet 1992;339:956-958. 
36. Morrison WI, Murray M, Whitelaw DD, Sayer PD. Pathology of infection with Trypanosoma brucei: disease syndromes in dogs and cattle resulting from severe tissue damage. Contrib Microbiol Immunol 1983;7:103-119.

37. Abbott NJ, Friedman A. Overview and introduction: the blood-brain barrier in health and disease. Epilepsia 2012;53(suppl 6):1-6.

38. Grab DJ, Nikolskaia O, Kim YV, et al. African trypanosome interactions with an in vitro model of the human blood-brain barrier. J Parasitol 2004;90:970-979.

39. Nikolskaia OV, de A Lima AP, Kim YV, et al. Blood-brain barrier traversal by African trypanosomes requires calcium signaling induced by parasite cysteine protease. J Clin Invest 2006;116:2739-2747.

40. Grab DJ, Garcia-Garcia JC, Nikolskaia OV, et al. Protease activated receptor signaling is required for African trypanosome traversal of human brain microvascular endothelial cells. PLoS Negl Trop Dis 2009;3:e479.

41. Frevert U, Movila A, Nikolskaia OV, et al. Early invasion of brain parenchyma by African trypanosomes. PLoS One 2012;7:e43913.

42. Laperchia C, Palomba M, Seke Etet PF, et al. Trypanosoma brucei invasion and T-cell infiltration of the brain parenchyma in experimental sleeping sickness: timing and correlation with functional changes. PLoS Negl Trop Dis 2016;10:e0005242.

43. Mulenga C, Mhlanga JD, Kristensson K, Robertson B. Trypanosoma brucei brucei crosses the blood-brain barrier while tight junction proteins are preserved in a rat chronic disease model. Neuropathol Appl Neurobiol 2001;27:77-85.

44. Philip KA, Dascombe MJ, Fraser PA, Pentreath VW. Blood-brain barrier damage in experimental African trypanosomiasis. Ann Trop Med Parasitol 1994;88:607-616.

45. Engelhardt B, Ransohoff RM. Capture, crawl, cross: the $T$ cell code to breach the blood-brain barriers. Trends Immunol 2012;33:579-589.

46. Kristensson K, Nygård M, Bertini G, Bentivoglio M. African trypanosome infections of the nervous system: parasite entry and effects on sleep and synaptic functions. Prog Neurobiol 2010;91:152-171.

47. Wolburg H, Mogk S, Acker S, et al. Late stage infection in sleeping sickness. PLoS One 2012;7:e34304.

48. Mogk S, Meiwes A, Shtopel S, et al. Cyclical appearance of African trypanosomes in the cerebrospinal fluid: new insights in how trypanosomes enter the CNS. PLoS One 2014;9:e91372.

49. Rodgers J, Jones A, Gibaud S, et al. Melarsoprol cyclodextrin inclusion complexes as promising oral candidates for the treatment of human African trypanosomiasis. PLoS Negl Trop Dis 2011;5:e1308.
50. Masocha W, Robertson B, Rottenberg ME, Mhlanga J, Sorokin L, Kristensson K. Cerebral vessel laminins and IFN-gamma define Trypanosoma brucei brucei penetration of the blood-brain barrier. J Clin Invest 2004;114:689-694.

51. Drennan MB, Stijlemans B, Van Den AJ, et al. The induction of a type 1 immune response following a Trypanosoma brucei infection is MyD88 dependent. J Immunol 2005; 175:2501-2509.

52. Amin DN, Vodnala SK, Masocha W, Sun B, Kristensson K, Rottenberg ME. Distinct Toll-like receptor signals regulate cerebral parasite load and interferon alpha/beta and tumor necrosis factor alpha-dependent T-cell infiltration in the brains of Trypanosoma brucei-infected mice. J Infect Dis 2012;205:320-332.

53. Sternberg JM, Forrest CM, Dalton RN, et al. Kynurenine pathway activation in human African trypanosomiasis. J Infect Dis 2017;215:806-812.

54. Rodgers J, Stone TW, Barrett MP, Bradley B, Kennedy PG. Kynurenine pathway inhibition reduces central nervous system inflammation in a model of human African trypanosomiasis. Brain 2009;132:1259-1267.

55. González A, Varo N, Alegre E, Díaz A, Melero I. Immunosuppression routed via the kynurenine pathway: a biochemical and pathophysiologic approach. Adv Clin Chem 2008;45:155-197.

56. Sternberg JM, Rodgers J, Bradley B, Maclean L, Murray M, Kennedy PG. Meningoencephalitic African trypanosomiasis: Brain IL-10 and IL-6 are associated with protection from neuro-inflammatory pathology. J Neuroimmunol 2005;167: 81-89.

57. Rodgers J, Bradley B, Kennedy PG, Sternberg JM. Central nervous system parasitosis and neuroinflammation ameliorated by systemic IL-10 administration in trypanosoma brucei-infected mice. PLoS Negl Trop Dis 2015;9:e0004201.

58. Agrawal S, Anderson P, Durbeej M, et al. Dystroglycan is selectively cleaved at the parenchymal basement membrane at sites of leukocyte extravasation in experimental autoimmune encephalomyelitis. J Exp Med 2006;203:1007-1019.

59. Zeni P, Doepker E, Schulze-Topphoff U, Huewel S, Tenenbaum T, Galla HJ. MMPs contribute to TNF-alpha-induced alteration of the blood-cerebrospinal fluid barrier in vitro. Am J Physiol Cell Physiol 2007;293:C855-C864.

60. Masocha W, Rottenberg ME, Kristensson K. Minocycline impedes African trypanosome invasion of the brain in a murine model. Antimicrob Agents Chemother 2006; 50:1798-1804. 


\title{
Neurology \\ Neuroimmunology \& Neuroinflammation
}

\author{
Generation of neuroinflammation in human African trypanosomiasis \\ Jean Rodgers, Israel Steiner and Peter G. E Kennedy \\ Neurol Neuroimmunol Neuroinflamm 2019;6; \\ DOI 10.1212/NXI.0000000000000610
}

This information is current as of August 29, 2019

Updated Information \&

Services

References

Permissions \& Licensing

Reprints including high resolution figures, can be found at:

http://nn.neurology.org/content/6/6/e610.full.html

This article cites 56 articles, 7 of which you can access for free at: http://nn.neurology.org/content/6/6/e610.full.html\#\#ref-list-1

Information about reproducing this article in parts (figures,tables) or in its entirety can be found online at:

http://nn.neurology.org/misc/about.xhtml\#permissions

Information about ordering reprints can be found online: http://nn.neurology.org/misc/addir.xhtml\#reprintsus

Neurol Neuroimmunol Neuroinflamm is an official journal of the American Academy of Neurology.

Published since April 2014, it is an open-access, online-only, continuous publication journal. Copyright

Copyright (C) 2019 The Author(s). Published by Wolters Kluwer Health, Inc. on behalf of the American

Academy of Neurology.. All rights reserved. Online ISSN: 2332-7812.

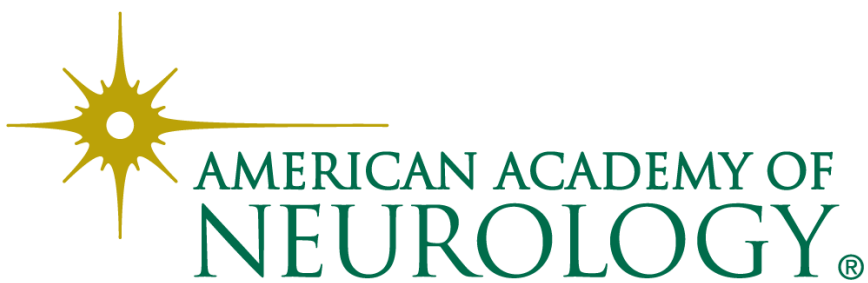

